\title{
The Use of Neural Network Analysis of Brain 18F-FDG PET in Diagnosis of Dementia Subjects
}

\section{Eric S. K. See, David Wai Chow Yeung}

Department of Diagnostic Radiology, Li Ka Shing Faculty of Medicine, The University of Hong Kong, Hong Kong, China

Correspondence to: Eric S. K. See, ericsees@gmail.com; David W. C. Yeung, yeungdw@gmail.com Keywords: Dementia, Artificial Neural Network, CortexID, PET-CT

Received: January 4, 2019 Accepted: February 22, $2019 \quad$ Published: February 25, 2019

Copyright $\odot 2019$ by authors and Scientific Research Publishing Inc.

This work is licensed under the Creative Commons Attribution International License (CC BY 4.0).

http://creativecommons.org/licenses/by/4.0/

\section{(c) (1) Open Access}

\section{ABSTRACT}

Since the world population is aging rapidly, the prevalence of dementia is also rising rapidly thus causing a great impact on individuals, families and societies. Accurate classification and level measurement of dementia are very importance in the disease management. Numerous studies show that ${ }^{18} \mathrm{~F}$-FDG-brain scan can differentiate various types of dementia. However, correct and accurate interpretation of nuclear images requires physicians who are well experienced. Therefore, it is worthwhile to build an automatic diagnostic system for it. In this paper, we present a novel method by using an artificial neural network (ANN) to analyze CortexID of brain PET-CT scan with clinical and laboratory data for dementia classification. Moreover, the ANN was trained to indicate the clinical severity of the disease as reflected by MMSE score. All ANNs were trained and tested again with an experienced physician's seventy diagnosis and the results were very promising. The dementia classifier achieved $96 \%$ accuracy and the mapper network could correctly predict the MMSE score with 0.782 regression value.

\section{INTRODUCTION}

The Hong Kong population is aging rapidly such that the elderly population (those aged 65 years or older) is estimated to increase from approximately $13 \%$ in 2009 to $28 \%$ by 2039 , there will be 2.5 million elder people in Hong Kong [1]. Since dementia is an age-related condition, the prevalence of dementia is expected to rise rapidly, and the consequent has an extensive impact on individuals, families, health and social welfare systems [2]. The number of dementia cases is projected to increase from 85,012 elder people in 2009 to 271,320 people in 2039. This is an important issue to be tackled and a dementia strategy for Hong Kong needs to be formulated. In order to cope with it, one direction is to develop accurate diagnostic tools, particular at early stage when treatment outcomes are most effective. Nuclear imaging and bio- 
markers diagnosis are the promising approach.

\subsection{Definition of Dementia}

Dementia is a group of diseases that primarily affect older people; but it is not a normal part of aging. The World Health Organization's International Classification of Disease (1992-1994) defines dementia as:

"Dementia is a syndrome due to disease of the brain, usually of a chronic or progressive nature, in which there is disturbance of multiple higher cortical functions, including memory, thinking, orientation, comprehension, calculation, learning capacity, language and judgment. The impairments of cognitive function are commonly accompanied, and occasionally preceded by deterioration in emotional control, social behavior, or motivation".

\subsection{Types and Causes of Dementia [3]}

There are numerous different forms of dementia and each has its own causes. Dementia can also be a symptom that develops in the later stages of other illnesses. The common types and causes of dementia include:

- Alzheimer's disease (AD) [4] accounts for 50\% - 70\% of all dementia. It is a progressive degeneration disease that shrinks brain cells, builds up tangles in soma and senile plaques, a neuritic process around an amyloid core, in extracellular space. These damage neurons connections disrupt messages within the brain.

- Vascular dementia $(\mathrm{VaD})[5]$ is the second $(15 \%-20 \%)$ most common form of dementia, which is caused by cerebrovascular conditions, usually caused by a series of small stroke. As the result, symptoms may not be noticeable or may only be temporary.

- Mixed dementia (MD) [6] is a condition of which pathologies of $\mathrm{AD}$ and $\mathrm{VaD}$ coexist. Around $45 \%$ of $\mathrm{AD}$ patients exhibit significant vascular pathology.

- Lewy bodies dementia (LBD) [7] is caused by abnormal microscopic deposits of spherical structuring Lewy bodies in the cerebral cortex.

- Frontotemporal Lobar Degeneration (FTLD) [8] damages the cells in the frontal lobes, anterior temporal lobes or both, giving rise to a spectrum of dementing disorders. It has a high familial incidence with approximately $40 \%$ of patients having a similar disorder history in a first degree-relative.

- Mild Cognitive Impairment (MCI) $[9,10]$ is considered as a prodromal stage of dementia, with noticeable cognitive changes, but no significant disability to interfere with daily life.

\subsection{Assessments of Dementia [11]}

There is no single diagnostic test for all dementias and a comprehensive assessment (such as clinical history taking, cognitive and physical examination, medication review, brain scanning, etc.) is required in order to exclude treatable causes of cognitive impairment.

Common assessments include:-

- Mini-mental state examination (MMSE), using in initial checkup, to analyze a subject's cognitive status. It uses a set of questions to provide measures of orientation, registration, short-term memory, motor and language functioning.

- Routine heametology and biochemistry tests to detect untreated syphilis, abnormality of thyroid function and decline of B12.

- MRI/CT brain scan to show the level of any atrophy and any vascular disease or infarcts.

However, absolute confirmation of the diagnosis can only be made by a biopsy and an autopsy of brain tissues.

\section{2. ${ }^{18}$ F-FDG PET-CT BRIAN SCAN AND CORTEX-ID}

Numerous studies have found that $\mathrm{AD}$ and other dementias could alter brain metabolisim significantly. AD patients usually present the posterior cingulate and parietotemporal cortices hypometabolisim 
and spread into the frontal lobes in advanced stage. In contrast, FTLD patients show manifestly hypometabolism in the frontal and anterior temporal cortices, while DLB patients present hypometabolism in the posterior brain comprising primarily the parietoocipital regions. Theoretically, ${ }^{18} \mathrm{~F}$-FDG PET scan can help in early diagnosis of $\mathrm{AD}$ and other dementias by highlighting these decreased FDG uptake cortex regions before MRI or CT scans can detect any structural brain damage.

To quantify the cortex metabolic activity, the reconstructed FDG PET images of the patient are processed with CortexID, developed by Dr. Satoshi Minoshima [12,13]. The software provides automated brain assessment through quantification of the comparison of local peak activity values, at standardized anatomical locations, with the corresponding reference normal peak activity in age-stratified control subjects. 3D stereotactic surface projections of the brain are generated and 31 regional Z-scores are calculated. A positive Z-score is given in a hypometabolic CortexID area.

By using these 31 regional Z-scores with MRI cerebrovascular condition, two ANNs were designed for dementias differentiation and MMSE score mapping. The details will be presented in section 4 .

\section{MULTILAYER PERCEPTRON (MLP) [14-16]}

ANN is a mathematical model to simulate the structure and functionalities of biological neural networks. The basic building block, artificial neuron, consists of multiplying every input value with individual weight, summing all weighted values and activating through the activation function (Figure 1 and Figure 2). Its mathematical description is:

$$
y(k)=g\left(\sum_{i=1}^{m} w_{i}(k) \cdot x_{i}(k)+b\right)
$$

where $x_{i}(k), w_{i}(k)$ and $y(k)$ are input, weight and output values in discrete time $k$ respectively,

$b$ is bias,

$g$ is a activation function.

A multilayer perceptron (Figure 3) is a feedforward artificial neural network and consists at least of three layers of nodes (artifical neuron): an input layer, a hidden layer and an output layer. It can divide the multi-dimensional feature space into arbitrartly complex decision regions and represent any boolean function.

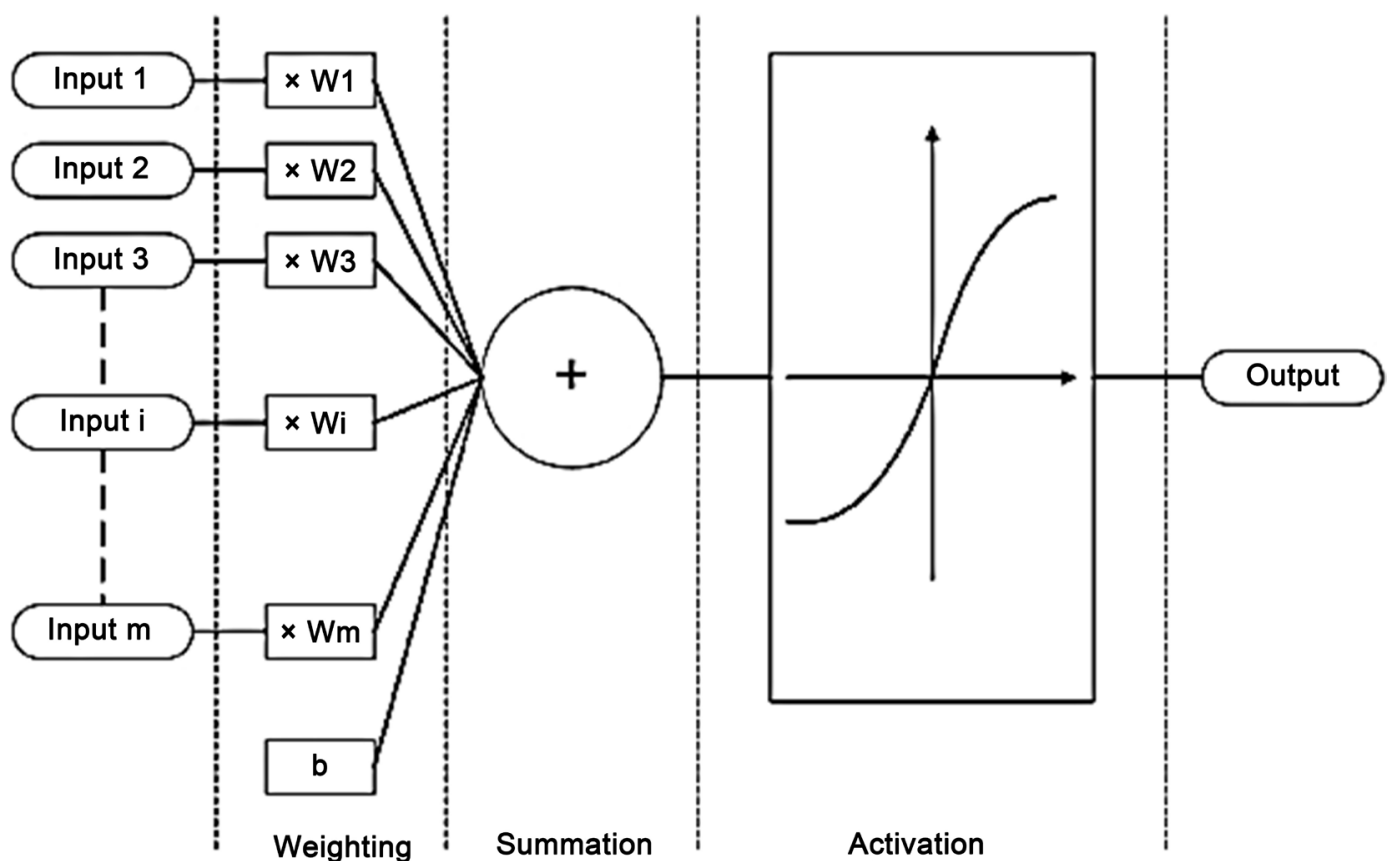

Figure 1. Working principle of an artifical neuron. 


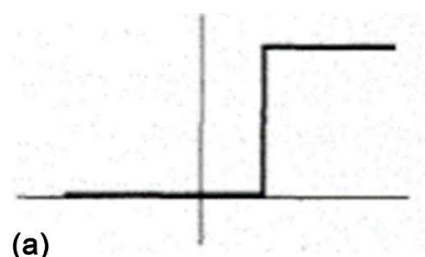

(a)

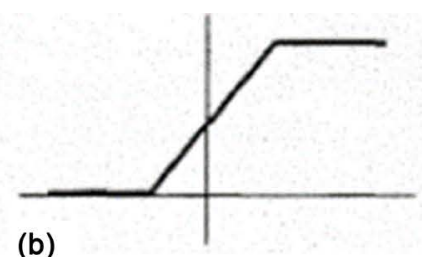

(b)

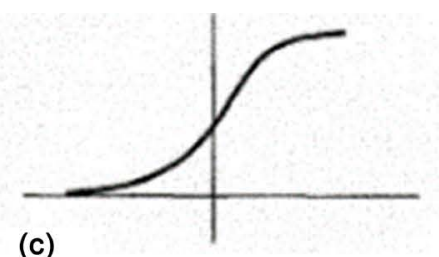

(c)

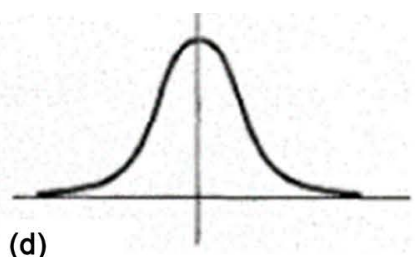

(d)

Figure 2. Different types of activation functions: (a) Threshold, (b) Piecewise linear, (c) Sigmoid, and (d) Gaussian.

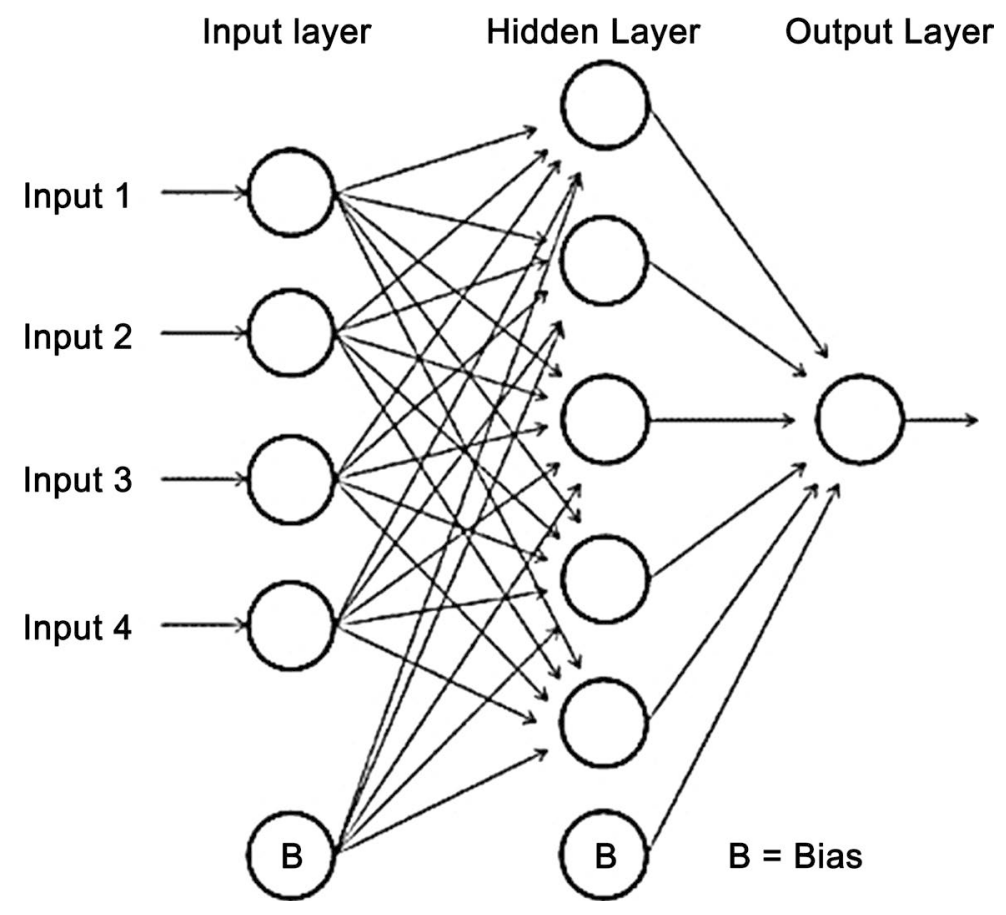

Figure 3. Multilayer perceptron.

Therefore MLP is widely and successfully used for pattern classification.

MLP utilizes the backpropagation rule for training. The rule repetitively calculates an error function for each input and backpropagates the error from one layer to the previous one. The weights are adjusted in direct proportional to the error in the nodes to which it is connected. The back-propagation algorithm is described below:

Step:

1) Initialize the weights randomly.

2) Arbitrarily select an input $X$.

3) Feed the signal through the network.

4) Compute $\delta_{i}^{L}$ in the output layer

$$
\delta_{i}^{L}=g^{\prime}\left(h_{i}^{L}\right)\left(d_{i}-y_{i}^{L}\right)
$$

where $h_{i}^{L}$ is the net input to the $I^{\text {th }}$ neuron in the $L^{\text {th }}$ layer,

$g^{\prime}$ is the derivative of the activation function,

$d_{i}$ and $y_{i}^{L}$ are the desired and feed forward output respectively.

5) Back-propagate the errors and compute the preceding layers deltas

$$
\delta_{i}^{l}=g^{\prime}\left(h_{i}^{l}\right) \sum_{j} w_{i j}^{l+1} \delta_{j}^{l+1}, l=(L-1), \cdots, 1
$$


6) Update weights

$$
\Delta w_{i j}^{l}=\eta \delta_{i}^{l} y_{i}^{l} \text { where } \eta \in(0,1)
$$

7) Repeat step 2 until the error in the output layer is below a prespecified values.

\section{BUILDING NNS OF USING CORTEXID TO MODEL PHYSICIAN'S DIAGNOSIS AND MMSE SCORE MAPPING}

MLPs are universal function approximators so they can be used to create mathematical models for function mapping as well as classifier. Therefore, MLPs were chosen in our application. Since too much hidden layers results in memorizing and not learning, one hidden layer is sufficient for the tasks. Except linear output neurons were used in MMSE score mapping, all neurons employ sigmoid activition function and trained with back-propagation algorithm.

\subsection{Data Preparation}

108 PET-CT examination radiological reports from University of Hong Kong at Queen Mary Hospital were screened. 87 reports are dementia cases and 17 of them were excluded because of incomplete CortexID data or unclassified or only having a single incidence. Moreover, 55 reports have MMSE scores and the frequency distribution of dementia is tabulated in Table 1.

It is observed that the distribution is severe skewed which will provide poor accuracies in most ANNs. To cope with the problems, class distributions need to be normalized by creating synthetic samples with Z-scores perturbed randomly [17]. Since VaD can only be accurately detected by MRI/CT imaging, the cerebrovascular condition becomes one of the input attributes of neural network in addition with 31 CortextID Z-scores.

Table 1. The dementia distribution in the dataset for classifer training.

\begin{tabular}{cc}
\hline Type of Dementia & Number of cases \\
\hline MCI & 13 \\
AD & 12 \\
VaD & 12 \\
MD & 27 \\
LBD & 6 \\
\hline
\end{tabular}

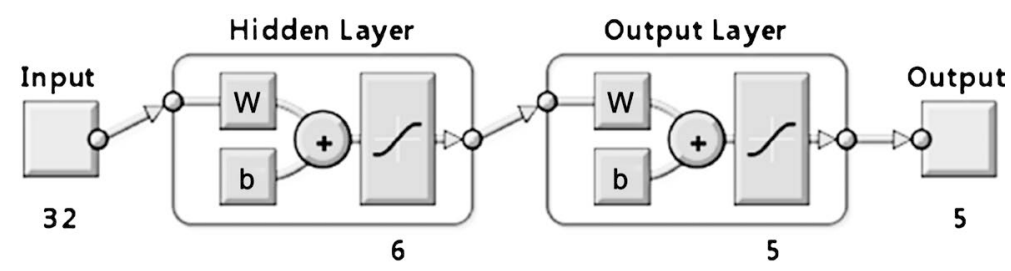

Figure 4. Dementia Classifier.

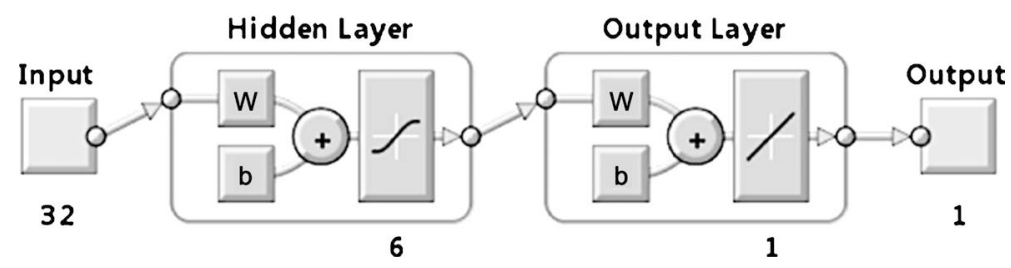

Figure 5. MMSE Score Mapper. 


\subsection{Training Procedure}

We used Matlab 2010 neural toolbox to build the ANNs. The data was divided randomly into the training set, the validation set and the test set. The training set is used for computing the gradient and updating the network weights. The validation set is used to improve generalization and to stop training when the network starts to overfit the data. The test set error is for different models comparison. For the dementia classifier training, the data is partitioned with a $60 \%$ training set, $20 \%$ validation set and $20 \%$ test set. In MMSE score mapping, the division portions were adjusted to $70 \%, 10 \%$ and $20 \%$ respectively.

\subsection{Trained NN Models}

In order to determine the optimum number of hidden neurons, the amount of neurons in the hidden layer is progressive increasing until the errors of the training, validation and test data sets are below the prespecified threshold.

The obtained NN models for dementia classifier (Figure 4) and MMSE score mapper (Figure 5) are shown.

\section{RESULTS}

\subsection{Dementia Classifier}

The performance of the classifier can be visualized in the confusion matrices (Figure 6(a)). 7.4\% of $\mathrm{AD}$ and $\mathrm{VaD}$ cases were misclassified as $\mathrm{MCI}$ and $\mathrm{MD}$ respectively. Moreover, since the ROC curves (Figure 6(b))

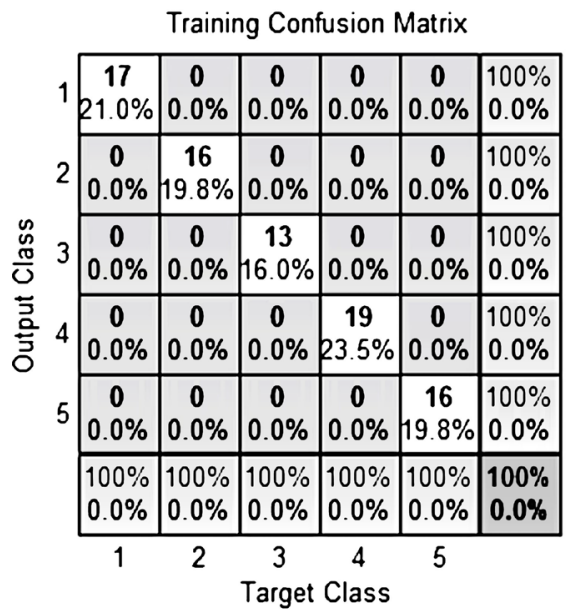

Test Confusion Matrix

\begin{tabular}{|c|c|c|c|c|c|c|}
\hline \multirow{4}{*}{ 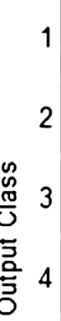 } & $\begin{array}{c}\mathbf{4} \\
14.8 \%\end{array}$ & $\begin{array}{c}1 \\
3.7 \%\end{array}$ & $\begin{array}{c}0 \\
0.0 \%\end{array}$ & $\begin{array}{c}0 \\
0.0 \%\end{array}$ & $\begin{array}{c}0 \\
0.0 \%\end{array}$ & $\begin{array}{l}30.0 \% \\
20.0 \%\end{array}$ \\
\hline & $\begin{array}{c}0 \\
0.0 \%\end{array}$ & $\begin{array}{c}5 \\
18.5 \%\end{array}$ & $\begin{array}{c}0 \\
0.0 \%\end{array}$ & $\begin{array}{c}0 \\
0.0 \%\end{array}$ & $\begin{array}{c}0 \\
0.0 \%\end{array}$ & $\begin{array}{l}100 \% \\
0.0 \%\end{array}$ \\
\hline & $\begin{array}{c}0 \\
0.0 \%\end{array}$ & $\begin{array}{c}0 \\
0.0 \%\end{array}$ & $\begin{array}{c}7 \\
25.9 \%\end{array}$ & $\begin{array}{c}0 \\
0.0 \%\end{array}$ & $\begin{array}{c}0 \\
0.0 \%\end{array}$ & $\begin{array}{l}100 \% \\
0.0 \%\end{array}$ \\
\hline & \begin{tabular}{|c|}
0 \\
$0.0 \%$
\end{tabular} & $\begin{array}{c}0 \\
0.0 \%\end{array}$ & $\begin{array}{c}1 \\
3.7 \%\end{array}$ & $\begin{array}{c}1 \\
3.7 \%\end{array}$ & $\begin{array}{c}0 \\
0.0 \%\end{array}$ & $\begin{array}{l}50.0 \% \\
50.0 \%\end{array}$ \\
\hline 5 & $\begin{array}{c}0 \\
0.0 \%\end{array}$ & $\begin{array}{c}0 \\
0.0 \%\end{array}$ & $\begin{array}{c}0 \\
0.0 \%\end{array}$ & $\begin{array}{c}0 \\
0.0 \%\end{array}$ & $\begin{array}{c}8 \\
29.6 \%\end{array}$ & $\begin{array}{l}100 \% \\
0.0 \%\end{array}$ \\
\hline & $\begin{array}{l}100 \% \\
0.0 \%\end{array}$ & $\begin{array}{l}33.3 \% \\
16.7 \%\end{array}$ & $\begin{array}{l}37.5 \% \\
12.5 \%\end{array}$ & $\begin{array}{l}100 \% \\
0.0 \%\end{array}$ & $\begin{array}{l}100 \% \\
0.0 \%\end{array}$ & $\begin{array}{l}92.6 \% \\
7.4 \%\end{array}$ \\
\hline & 1 & 2 & 3 & 4 & 5 & \\
\hline
\end{tabular}

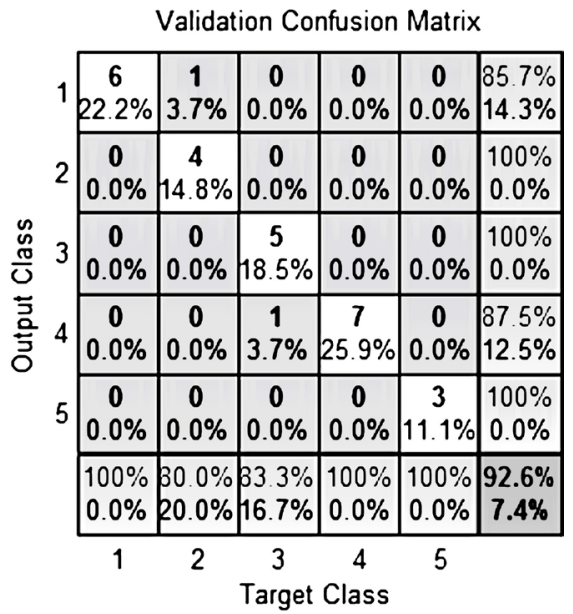

All Confusion Matrix

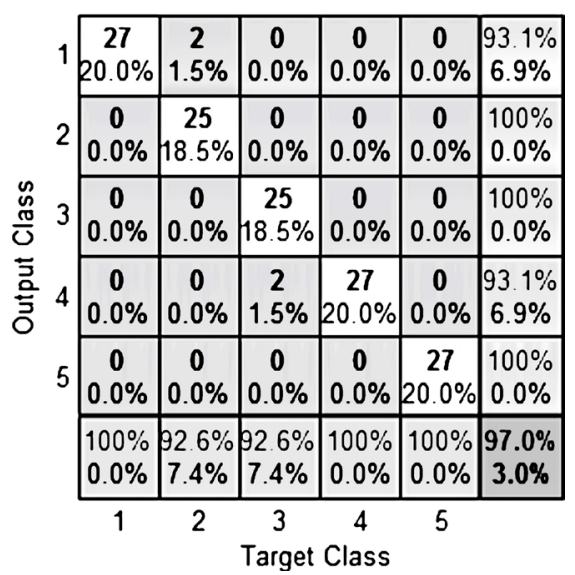

(a) 


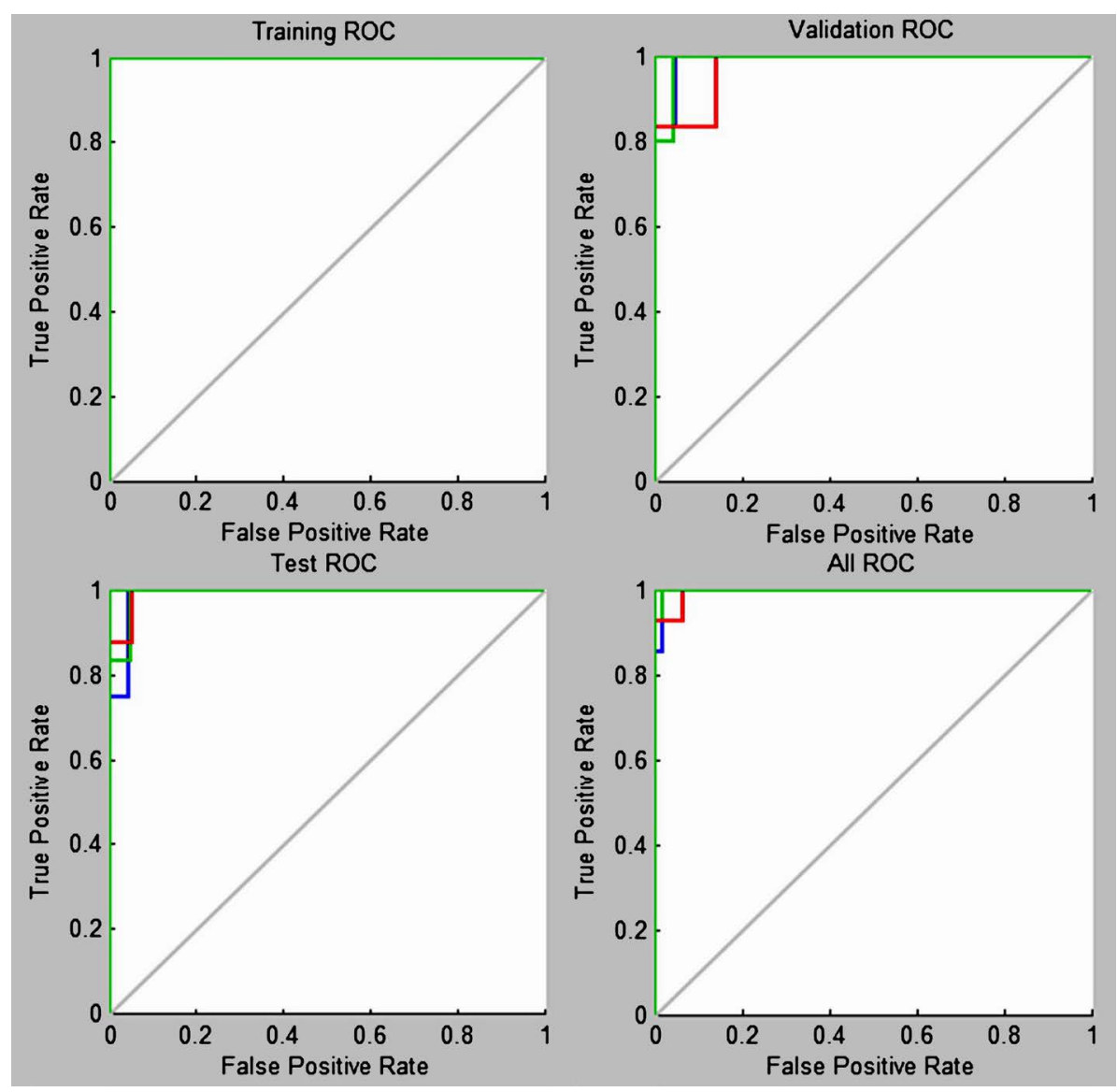

(b)

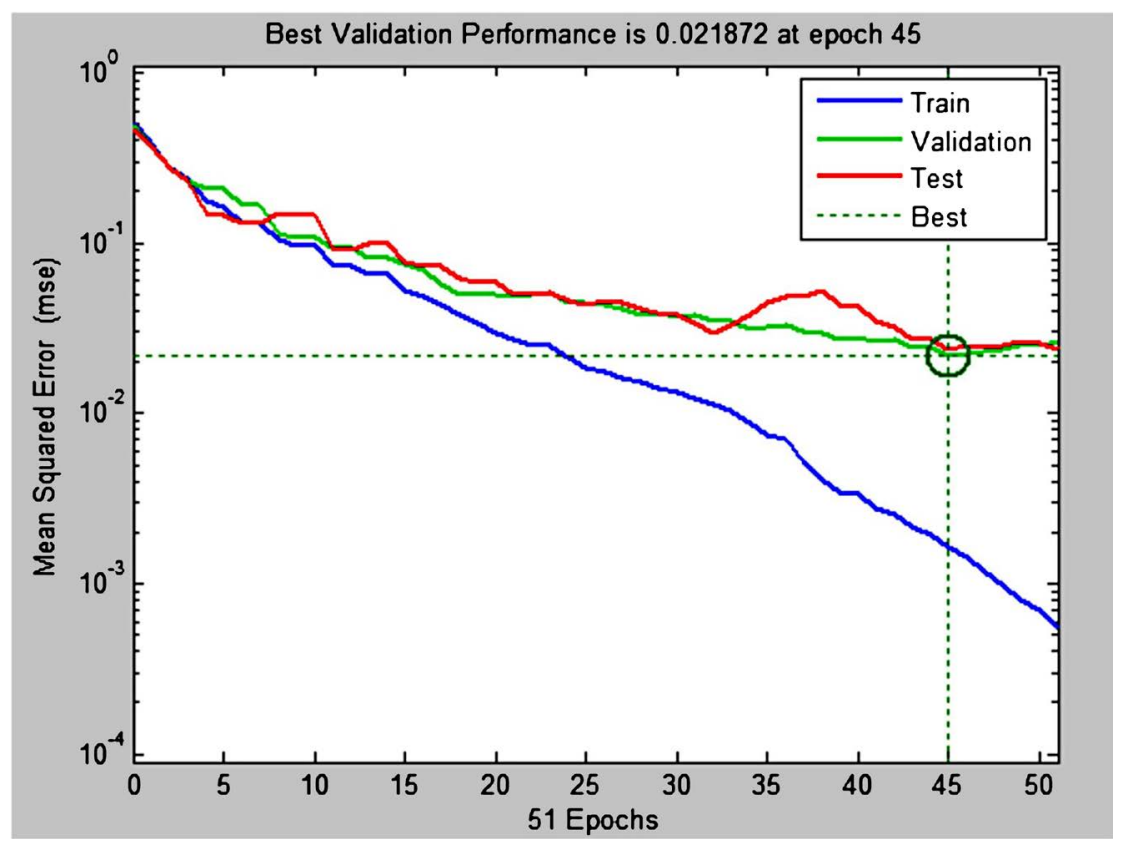

(c)

Figure 6. (a) The confusion matrices [1-MCI, 2-AD, 3-VaD, 4-MD, 5-LBD]; (b) the receiver-operating characteristics and (c) mean squared error of the dementia classifier. 
are very close to the upper left corner, this implies the overall accuracy of the classifier is very high. Figure 6(c) reflects that the network was continued adjusting the weights to classify the data pattern and the best validation performance was obtained at epoch 45 with mean square error 0.021872 .

\subsection{MMSE Score Mapping}

The correlations between the outputs of mapping function network and desired MMSE target values were plotted in Figure 7. The root means squared errors between output and target of training, validation and testing datasets are 1.11, 1.74 and 6.62 respectively. An outlier occurred in the test-set upshifts the regression line significantly. Moreover, the overall regression R-value is 0.87 .

\section{PERFORMANCE ANALYSIS}

To prevent biased, untrained data sets are used to calculate the sensitivities and specificities of the dementia classifier. Other related performance indices are also computed and tabulated in Table 2.

Based on the analysis table, it is observed that the dementia classifier was learnt to categorise different types of dementia very accurately as a radiologist by using 31 regional CortexID Z-scores with cerebrovascular condition.

In MMSE score mapping study, the sample sizes of validation and testing data sets are 6 and 11; their regression values are 0.983 and 0.646 . Therefore, the combined regression value is

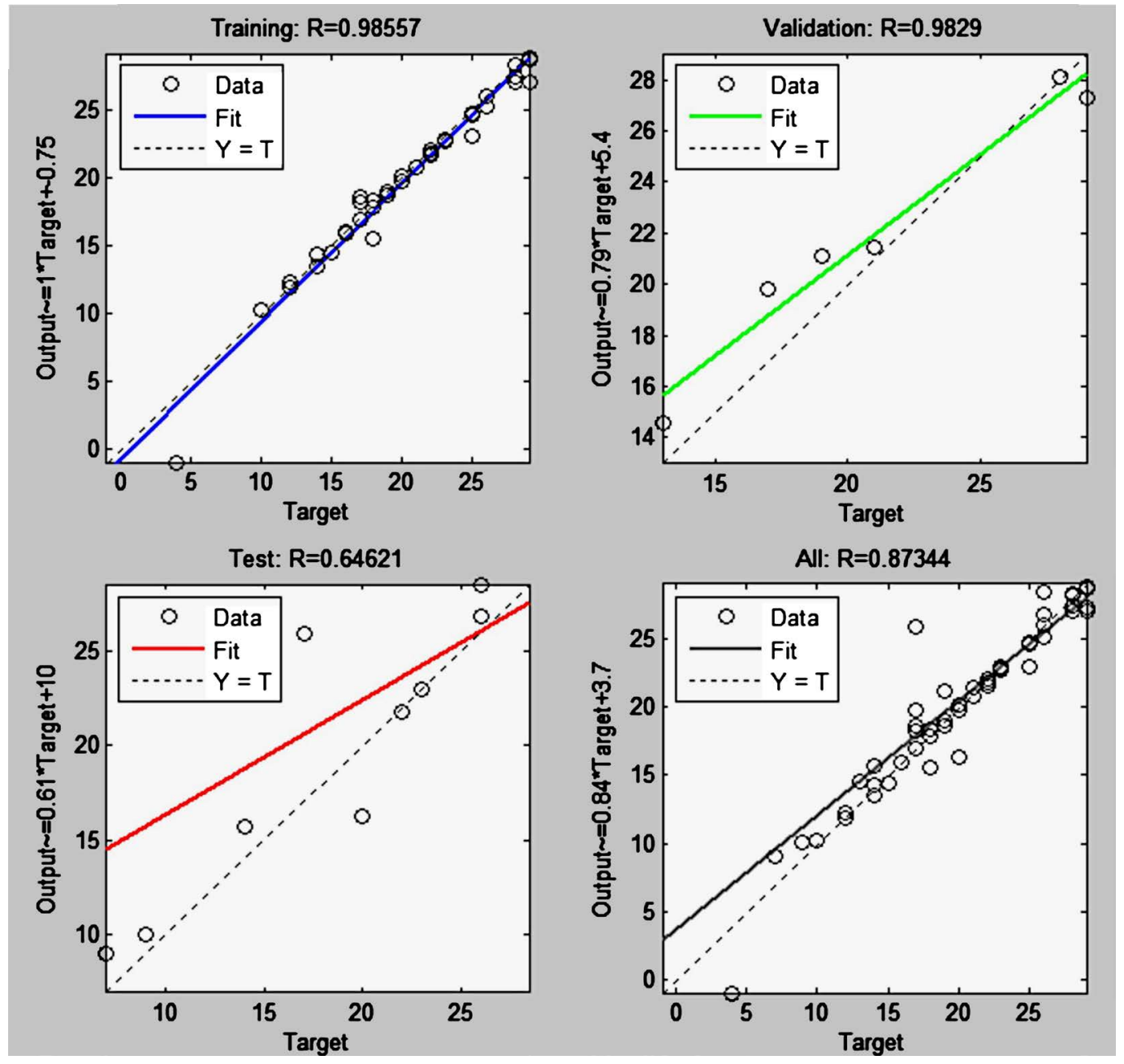

Figure 7. Regression Plots between MMSE Mapping Function outputs and targets. 
Table 2. Performance Analysis of Dementia Classifier on the Untrained Data Set (Validation + Test).

\begin{tabular}{|c|c|c|c|c|c|c|c|c|c|}
\hline Dementia & $\begin{array}{c}\text { True } \\
\text { Positive }\end{array}$ & $\begin{array}{c}\text { False } \\
\text { Positive }\end{array}$ & $\begin{array}{c}\text { True } \\
\text { Negative }\end{array}$ & $\begin{array}{c}\text { False } \\
\text { Negative }\end{array}$ & Sensitivity & Specificity & PPV & NPV & Accuracy \\
\hline MCI & 10 & 2 & 42 & 0 & $100 \%$ & $95.5 \%$ & $83.3 \%$ & $100 \%$ & $96.3 \%$ \\
\hline $\mathrm{AD}$ & 9 & 0 & 43 & 2 & $81.8 \%$ & $100 \%$ & $100 \%$ & $95.6 \%$ & $96.3 \%$ \\
\hline $\mathrm{VaD}$ & 12 & 0 & 40 & 2 & $85.7 \%$ & $100 \%$ & $100 \%$ & $95.2 \%$ & $96.3 \%$ \\
\hline $\mathrm{MD}$ & 8 & 2 & 44 & 0 & $100 \%$ & $95.7 \%$ & $80 \%$ & $100 \%$ & $96.3 \%$ \\
\hline DLB & 11 & 0 & 43 & 0 & $100 \%$ & $100 \%$ & $100 \%$ & $100 \%$ & $100 \%$ \\
\hline Dementia & \multicolumn{3}{|c|}{ Type I Error } & \multicolumn{3}{|c|}{ Type II Error } & \multicolumn{3}{|c|}{ Area under ROC curve (AUC) } \\
\hline MCI & \multicolumn{3}{|c|}{$4.5 \%$} & \multicolumn{3}{|c|}{$0 \%$} & \multicolumn{3}{|c|}{0.985} \\
\hline $\mathrm{AD}$ & \multicolumn{3}{|c|}{$0 \%$} & \multicolumn{3}{|c|}{$18.2 \%$} & \multicolumn{3}{|c|}{0.979} \\
\hline $\mathrm{VaD}$ & \multicolumn{3}{|c|}{$0 \%$} & \multicolumn{3}{|c|}{$14.3 \%$} & \multicolumn{3}{|c|}{0.981} \\
\hline $\mathrm{MD}$ & \multicolumn{3}{|c|}{$4.3 \%$} & \multicolumn{3}{|c|}{$0 \%$} & \multicolumn{3}{|c|}{0.985} \\
\hline DLB & \multicolumn{3}{|c|}{$0 \%$} & \multicolumn{3}{|c|}{$0 \%$} & \multicolumn{3}{|c|}{1} \\
\hline
\end{tabular}

$$
R_{\text {validation+testing }}=\sqrt{\frac{6 \times 0.983^{2}+11 \times 0.646^{2}}{6+11}}=0.782
$$

Since the $R_{\text {validation+testing }}$ is very close to 1 , the mapping network correlates highly with MMSE score.

\section{CONCLUSIONS}

Two multilayer perceptrons, based on 31 regional CortexID Z-scores, were designed and trained to classify different types of dementia and map with MMSE score. Comparing with physicians diagnosis, the dementia classifier achieved $96 \%$ accuracy. The mapper network can correctly predict the MMSE score with 0.782 regression value.

However, due to the limit number of records, we do not know whether the network can be trained to differentiate other types of dementias, such as frontotemporal dementia, Parkinsonian syndromes, etc. Further study and verification are required.

\section{ACKNOWLEDGEMENTS}

This is a retrospective chart review study and is supported by the Department of Diagnostic Radiology, University of Hong Kong and the Hospital Authority, Hong Kong. We also wish to acknowledge the clinical data support of Prof. Leung-Wing Chu of the Department of Medicine of the University of Hong Kong.

\section{CONFLICTS OF INTEREST}

The authors declare no conflicts of interest regarding the publication of this paper.

\section{REFERENCES}

1. Hong Kong Population Projection 2010-2039.

https://www.statistics.gov.hk/pub/B1120015042010XXXXB0100.pdf 
2. Yu, R., Chau, P.H., McGhee, S.M., Cheung, W.L., Chan, K.C., Cheung, S.H. and Woo, J. (2012) Trends in Prevalence and Mortality of Dementia in Elderly Hong Kong Population: Projections, Disease Burden, and Implications for Long-Term Care, International Journal of Alzheimer's Disease, 2012, Article ID 406852. https://doi.org/10.1155/2012/406852

3. Morris, J.C. (1996) Classification of Dementia and Alzheimer's Disease. Acta Neurologica Scandinavica, 165, 41-50. https://doi.org/10.1111/j.1600-0404.1996.tb05871.x

4. Bayer, T.A., Wirths, O., Majtényi, K., Hartmann, T., Multhaup, G., Beyreuther, K. and Czech, C. (2001) Key Factors in Alzheimer's Disease: $\beta$-Amyloid Precursor Protein Processing, Metabolism and Intraneuronal Transport. Brain Pathology, 11. https://doi.org/10.1111/j.1750-3639.2001.tb00376.x

5. Jellinger, K.A. (2013) Pathology and Pathogenesis of Vascular Cognitive Impairment: A Critical Update. Frontiers in Aging Neuroscience, 5, 17. https://doi.org/10.3389/fnagi.2013.00017

6. Custodio, N., Montesinos, R., Lira, D., Herrera-Pérez, E., Bardales, Y. and Valeriano-Lorenzo, L. (2017) Mixed Dementia: A Review of the Evidence. Dementia \& Neuropsychologia, 11, 364-370. https://doi.org/10.1590/1980-57642016dn11-040005

7. Mrak, R.E. and Griffin, W.S.T. (2007) Dementia with Lewy Bodies: Definition, Diagnosis, and Pathogenic Relationship to Alzheimer's Disease. Neuropsychiatric Disease and Treatment, 3, 619-625.

8. Seltman, R.E. and Matthews, B.R. (2012) Frontotemporal Lobar Degeneration: Epidemiology, Pathology, Diagnosis and Management. CNS Drugs, 26, 841-870. https://doi.org/10.2165/11640070-000000000-00000

9. Jonker, C. and Comijs, H.C. (2007) Mild Cognitive Impairment: A Prodromal Phase of Dementia? Tijdschr Gerontol Geriatr., 38, 115-121.

10. Bischkopf, J., Busse, A. and Angermeyer, M.C. (2002) Mild Cognitive Impairment: A Review of Prevalence, Incidence and Outcome According to Current Approaches. Acta Psychiatrica Scandinavica, 106, 403-414. https://doi.org/10.1034/j.1600-0447.2002.01417.x

11. Knopman, D.S., Boeve, B.F. and Petersen, R.C. (2003) Essentials of the Proper Diagnoses of Mild Cognitive Impairment. Dementia, and Major Subtypes of Dementia. Mayo Clinic Proceedings, 78, 1302. https://doi.org/10.4065/78.10.1290

12. Kakimoto, A., Kamekawa, Y., Ito, S., Yoshikawa, E., Okada, H.A., Nishizawa, S., Minoshima, S. and Ouch, Y. (2007) New Computer-Aided Diagnosis of Dementia Using Positron Emission Tomography: Brain Regional Sensitivity-Mapping Method. http://snmmi.files.cms-plus.com/docs/PCOENewsletter2007-2.pdf

13. Minoshima, S., Frey, K.S., Koeppe, R.A., Foster, N.L. and Kuhl, D.E. (1995) A Diagnostic Approach in Alzheimer's Disease Using Three-Dimensional Stereotactic Surface Projections of Fluorine-18-FDG PET. Journal of Nuclear Medicine, 36, 1238-1248.

14. Williams, J.A., Weakley, A., Cook, D.J. and Schmitter-Edgecombe, M. (2013) Machine Learning Techniques for Diagnostic Differentiation of Mild Cognitive Impairment and Dementia. Proceedings at the Twenty-Seventh AAAI Conference on Artificial Intelligence: Expanding the Boundaries of Health Informatics Using AI, Association for the Advancement of Artificial Intelligence, Bellevue, Washington, 14-18 July 2013, 71-76.

15. Patil, M.M. and Yardi, A.R. (2012) MLP Classifer for Dementia Levels. International Journal of Modeling and Optimization, 2, 567-569.

16. Defigueiredo, R.J.P., Shankle, W.R., Maccato, A., Dick, M.R., Mundkur, P., Mena, I. and Cotman, W.W. (1995) Neural-Network-Based Classification of Cognitively Normal, Demented, Alzheimer Disease and Vascular Dementia from Single Photon Emission with Computed Tomography Image Data from Brain. Proceedings of National Academy of Sciences of USA, 92, 5530-5534. https://doi.org/10.1073/pnas.92.12.5530

17. He, H. and Garcia, E.A. (2009) Learning from Imbalanced Data. IEEE Transactions on Knowledge and Data Engineering, 21, 1263-1284. 